\title{
Estudo de recobrimentos de silicato e aluminato obtidos por oxidação por plasma eletrolítico em liga de alumínio
}

\author{
Study of silicate and aluminate coatings \\ obtained by plasma electrolytic \\ oxidation of aluminum alloy
}

Bruna Almeida Barbosa ${ }^{1}$, Giselle Barata Costa ${ }^{2}$, Maria Eliziane Pires de Souza ${ }^{3}$

\footnotetext{
${ }^{1}$ Brintel Sistemas de Segurança, Av Presidente Vargas 1461, CEP: 35661- 000, Pará de Minas, Minas Gerais, Brasil.

${ }^{2}$ Instituto de Geociências e Engenharia, Universidade Federal do Sul e Sudeste do Pará, Marabá, Pará, Brasil.

${ }^{3}$ Engenharia Mecânica, Centro de Ciências Exatas e Tecnologia, Universidade Federal do Maranhão, São Luís, Maranhão, Brasil.

e-mail: brunna_barbosa@hotmail.com,gisamec@unifesspa.edu.br,maria.eliziane@ufma.br
}

\section{RESUMO}

As ligas de Alumínio são utilizadas para diversas finalidades e para a maioria dos casos a superfície desses materiais precisa ser modificada a fim de se melhorar propriedades como resistência ao desgaste e corrosão. Neste cenário, o processo de oxidação por plasma eletrolítico (PEO) surge como uma alternativa viável para produção de recobrimentos sobre esses substratos, já que o alumínio apresenta baixo ponto de fusão e este processo ocorre em baixas temperaturas. Neste trabalho, recobrimentos obtidos por plasma eletrolítico foram produzidos sobre a superfície de ligas de alumínio empregando diferentes parâmetros de forma a se comparar os recobrimentos obtidos. A caracterização foi feita por espectroscopia de impedância eletroquímica (EIE) com intuito de analisar o desempenho frente a corrosão. Modelo de circuito equivalente foi empregado para ajustar as curvas de EIE, de forma a melhor entender o comportamento da superfície. A partir dos resultados obtidos, foi possível constatar que em menor tensão $(250 \mathrm{~V})$, houve maior formação de filme sobre o substrato e consequentemente uma melhor proteção do mesmo. Quando comparado ao alumínio sem recobrimento, as amostras revestidas apresentaram uma melhora em termos de corrosão, apresentando maior resistência.

Palavras-chave: Alumínio, Corrosão, Plasma Eletrolítico, EIE.

\section{ABSTRACT}

Aluminum alloys are used for various purposes and in the most of cases the surface of such materials needs to be modified in order to improve properties such as wear resistance and corrosion. In this scenario, plasma electrolytic oxidation (PEO) process comes up as a viable alternative for the production of coatings on these substrates, since aluminum has low melting point and this process occurs at low temperatures. In this work, coatings obtained by PEO were produced on the surface of aluminum alloys, using different parameters in order to compare the properties of produced coatings. The coatings obtained were characterized by electrochemical impedance spectroscopy (EIS) in order to analyze the performance against corrosion. Equivalent circuit model was used to adjust the EIS curves, with a view to better understand the behavior of coatings. From the obtained results it was possible to verify that at lower voltage $(250 \mathrm{~V})$, there was a greater formation of film on the substrate and consequently a better protection of the same. When compared to uncoated aluminum, the coated samples presented an improvement of corrosion resistance.

Keywords: Aluminum, corrosion, electrolytic plasma, EIS.

\section{INTRODUÇÃO}

A modificação de superfícies geralmente é realizada nos materiais para melhorar propriedades como aparên- 
cia, resistência ao desgaste e corrosão, aderência entre outras [1-4]. Diversas técnicas de deposição têm sido utilizadas para criar coberturas com recobrimentos cerâmicos nas superfícies dos metais. Estes recobrimentos, que possuem elevada dureza e são bastante aderentes ao metal recoberto, garantem o aumento da resistência superficial ao desgaste das peças [5,6].

Para a obtenção de tais recobrimentos um processo que vem sendo bastante empregado é o de oxidação por plasma eletrolítico (PEO - Plasma electrolytic oxidation) [7-9]. Este tratamento pode ser aplicado tanto para limpeza da superfície quanto deposição de recobrimentos metálicos e/ou cerâmicos, dependendo da interação entre eletrólito, plasma e superfície [1]. Recobrimentos obtidos através do PEO conferem uma melhora nas superfícies dos metais e suas ligas em termos de resistência ao desgaste [10-12], à corrosão [1316] isolamento elétrico entre outros [17-19]. Vários trabalhos mostram que as propriedades dos recobrimentos dependem fortemente da natureza do substrato metálico [20-22], do tipo de fonte de potência [6,9], da densidade de corrente [13], composição e concentração do eletrólito [4,8,23].

O processo a plasma eletrolítico é ambientalmente limpo e aplica um potencial muito maior do que os processos de eletrodeposição convencionais, proporcionando a formação do plasma [24]. É um processo complexo, que envolve a combinação de processos parciais concorrentes: a formação do filme, sua dissolução e a formação de um dielétrico. A probabilidade do domínio de algum desses processos no processo geral, depende da natureza tanto do metal e do eletrólito, quanto do regime de corrente adotado. O último estágio do processo pode ser caracterizado como um estado semi-estacionário de micro descargas anódicas, que exibe uma progressiva mudança nas características durante a eletrólise [18, 24].

Neste trabalho, a superfície da liga de alumínio (AA 5052) foi modificada pela deposição de recobrimento obtido pelo processo de plasma eletrolítico, a partir de duas soluções eletrolíticas. Os recobrimentos foram avaliados por espectroscopia de impedância eletroquímica (EIE), empregando o ajuste por circuito equivalente para caracterizar o comportamento protetivo do filme.

\section{MATERIAIS E MÉTODOS}

Liga de Alumínio AA 5052 foi empregada como substrato. Antes do preparo dos recobrimentos as amostras foram limpas em cuba ultrassônica utilizando três etapas: banho com água e sabão, seguido de banho em água destilada e por fim banho em álcool isopropílico, com duração de 15 minutos cada banho, as amostras foram então secadas com soprador térmico.

A célula eletrolítica para o preparo dos recobrimentos é composta pela amostra de alumínio conectada ao anodo da fonte de corrente contínua (Modelo FCC 350-100i - Supplier), e posicionada entre os dois contra-eletrodos de aço inoxidável (catodo), agitador mecânico e a solução eletrolítica, como mostrado na Figura 1.

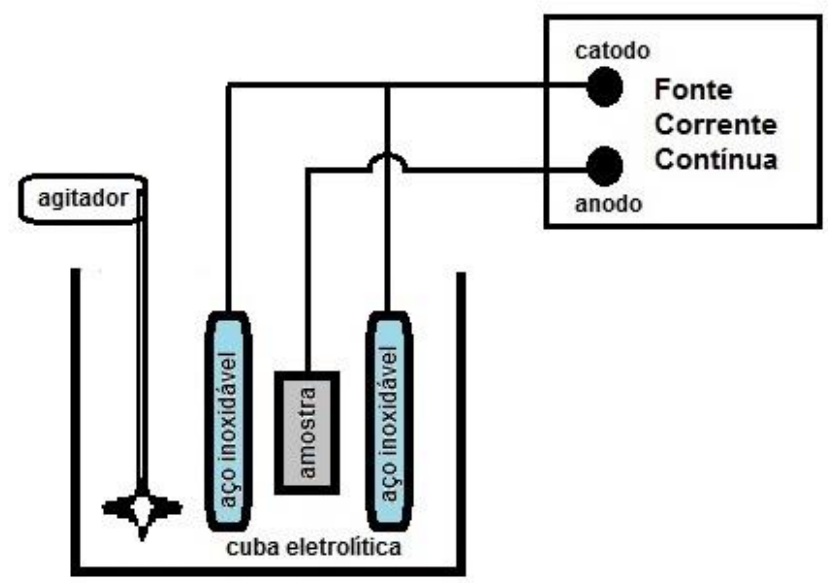

Figura 1: Desenho esquemático do aparato experimental utilizado para preparação dos recobrimentos

Para efeito de comparação foram empregadas duas soluções como meio eletrolítico para o preparo dos recobrimentos, na Tabela 1 são informadas as soluções e as suas respectivas concentrações. 
Tabela 1: Composição das soluções empregadas no PEO

\begin{tabular}{c|l}
\hline SOLUÇÃo & COMPOSIÇÃo \\
\hline 1 & Silicato de sódio $(10 \mathrm{~g} / \mathrm{l})+$ Fosfato de Sódio Bibásico $(1,5 \mathrm{~g} / \mathrm{l})$ \\
\hline 2 & Aluminato de sódio $(10 \mathrm{~g} / \mathrm{l})+$ Fosfato de Sódio Bibásico $(1,5 \mathrm{~g} / \mathrm{l})$ \\
\hline
\end{tabular}

Para a formação dos recobrimentos foram empregadas duas tensões finais $(250 \mathrm{~V}$ e $300 \mathrm{~V})$ com o intuito de comparar esses parâmetros. O aumento da tensão durante o processo de preparo dos recobrimentos foi realizado em intervalos de três minutos até se atingir o valor final de tensão, esse valor era então mantido por 15 minutos. Desta forma, todos os processos para a formação dos recobrimentos apresentaram um tempo total de 30 minutos. A temperatura e a corrente foram acompanhadas durante todo o processo. A denominação empregada para as amostras bem como suas respectivas condições de processo são apresentadas na Tabela 2 .

Tabela 2: Denominação das amostras e condições de processo

\begin{tabular}{c|c|c}
\hline AMOSTRA & SOLUÇÃO & TENSÃO FINAL (V) \\
\hline 1A & 1 & 250 \\
\hline 1B & 1 & 300 \\
\hline 2A & 2 & 250 \\
\hline 2B & 2 & 300 \\
\hline
\end{tabular}

As amostras foram pesadas antes e após o PEO, assim a quantidade de recobrimento foi especificada em termos de massa de filme formada.

A caracterização dos recobrimentos foi realizada por espectroscopia de impedância eletroquímica (EIE), utilizando o equipamento Potenciostato EG\&G 273A (Princeton Applied Research - PAR), acoplado a um amplificador lock-in, modelo 5210 (EG \& G), controlado pelo software Powersine®. A amplitude da tensão alternada aplicada foi de $10 \mathrm{mV}$ com frequências de $10 \mathrm{~Hz}$ a $0,01 \mathrm{~Hz}$. As medidas foram realizadas em potencial de circuito aberto. Foi utilizado uma célula eletroquímica equipada com três eletrodos, sendo a amostra o eletrodo de trabalho com $1 \mathrm{~cm}^{2}$, a platina o contra-eletrodo e $\mathrm{Ag} / \mathrm{AgCl}$ usado como eletrodo de referência. Como eletrólito foi utilizado $\mathrm{NaCl} 0,1$ molar.

\section{RESULTADOS E DISCUSSÃO}

A variação do corrente durante o processamento dos recobrimentos nas diferentes condições foi acompanhada e os resultados são apresentados na Figura 2.

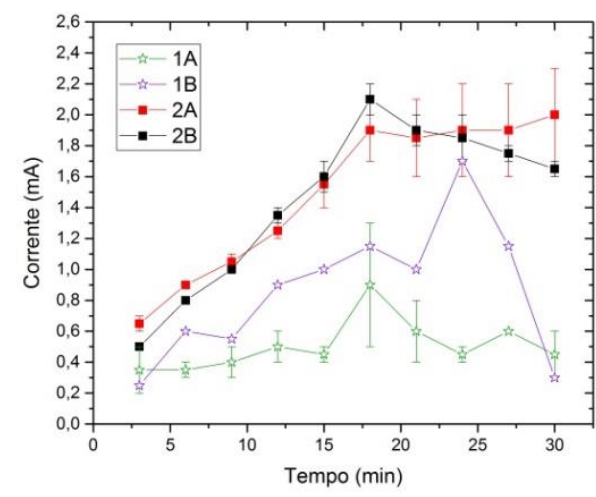

Figura 2: Variação da corrente em função do tempo do processo.

Como pode ser observado na Figura 2, para a solução 2 (aluminato/fosfato) a corrente apresenta au- 
mento praticamente linear à medida que a tensão vai sendo aumentada. Quando se atinge o valor final de tensão, após 15 minutos, a corrente chega a um valor máximo, passando a apresentar uma queda que continua até o final do processo. Esse comportamento pode ser representativo de um sistema com liberação de gás tanto na superfície do anodo quanto do catodo [18]. Para a solução 1 (silicato/fosfato), os valores de corrente atingidos foram menores, sendo que o aumento também se deu de forma menos expressiva, mas a luminescência característica do plasma $[18,24]$ pôde ser observada em tensões acima de $150 \mathrm{~V}$, para todos os procedimentos empregados. As diferenças no comportamento da corrente podem ser associadas ao eletrólito empregado e seu efeito sobre a superfície do substrato. Segundo Yerokhin [18] os eletrólitos podem tanto promover uma dissolução acelerada do substrato bem como ajudar na formação do filme passivo. Pelos resultados observados em termos de corrente e tensão, pode se dizer que a solução de aluminato favorece mais a dissolução que a formação de um filme, uma vez que os valores de corrente obtidos são mais elevados e pouco decrescem com a tensão final. Já a solução de silicato representaria uma maior tendência à formação de um filme passivo.

Um aumento na temperatura do eletrólito é esperado durante o processamento por plasma eletrolítico [18] e pode ser associado ao aumento tanto em tensão quanto em corrente necessários para a formação do plasma [2]. Como pode ser observado na Figura 3, a elevação que ocorre na temperatura do eletrólito durante o processamento em todas as condições estudas, não ultrapassou os $50{ }^{\circ} \mathrm{C}$, ficando dentro dos valores de temperatura de eletrólito observados em outros trabalhos [2], não havendo a necessidade de se fazer um resfriamento do banho durante o processo.

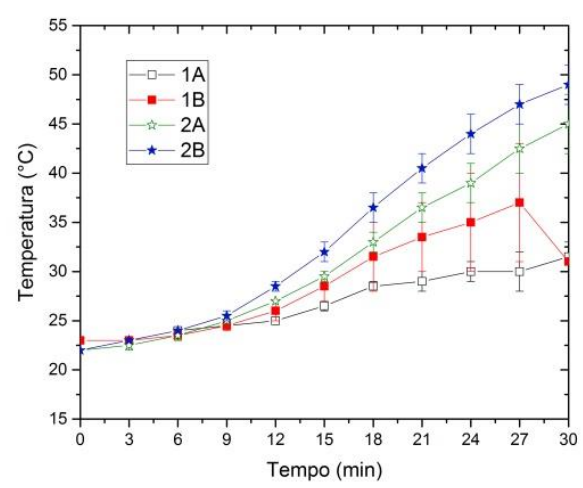

Figura 3: Variação da temperatura durante o processamento dos recobrimentos.

A quantidade de filme formado na superfície das amostras foi obtida através do peso das mesmas antes e após a formação dos recobrimentos e na Figura 4 são apresentados os resultados para as diferentes amostras estudadas. Como pode ser observado, os recobrimentos produzidos até 250 Volts resultaram em um maior aumento na massa das amostras, configurando uma quantidade maior de filme. A formação de menor quantidade de filme, para o procedimento até 300 Volts, independente da solução eletrolítica, pode ser relacionada a essa maior tensão, que leva naturalmente a uma destruição do recobrimento durante o processo ou uma formação de um recobrimento mais poroso [2,8]. Por outro lado, os baixos valores de corrente observados para a amostra 1A pode ter levado a formação de um filme mais compacto em relação aos outros, o que levou a uma variação maior na massa da amostra. Esse resultado pode ser explicado pelas condições de obtenção desses recobrimentos e dos fenômenos físicos envolvidos no processo [2].

As micro descargas que ocorrem durante o PEO são responsáveis pela fusão do filme juntamente com os elementos presentes no eletrólito, além disso, levam à formação de um recobrimento com porosidades e uma rugosidade característica [2,17]. Ao analisarmos a evolução da corrente, Figura 2, é possível dizer que os processos empregados nesse trabalho, não tenham atingido todos os estágios de formação, não permanecendo no estágio final que seria o de recristalização do filme óxido [8], fazendo com que os recobrimentos apresentem uma massa reduzida. Outro fato que pode ser apresentado para corroborar aos resultados foi o aparecimento de partículas em suspensão durante o processamento, Figura 5, sendo que à tensão de $250 \mathrm{~V}$ essas partículas só apareceram próximo aos 27 minutos e à $300 \mathrm{~V}$ estas já eram visíveis em apenas 15 minutos, o que também justificaria a menor quantidade de recobrimentos formado à $300 \mathrm{~V}$. 


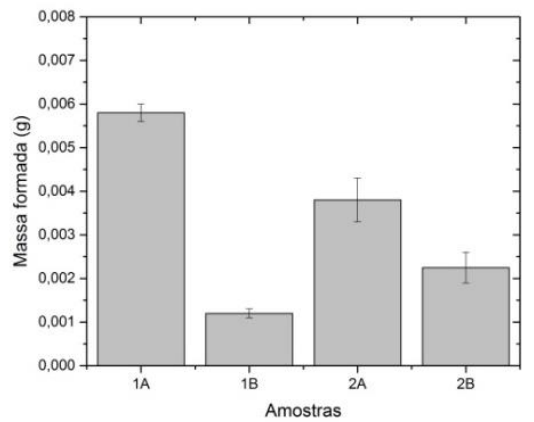

Figura 4: Massa de recobrimento formada para as diferentes condições de processo.

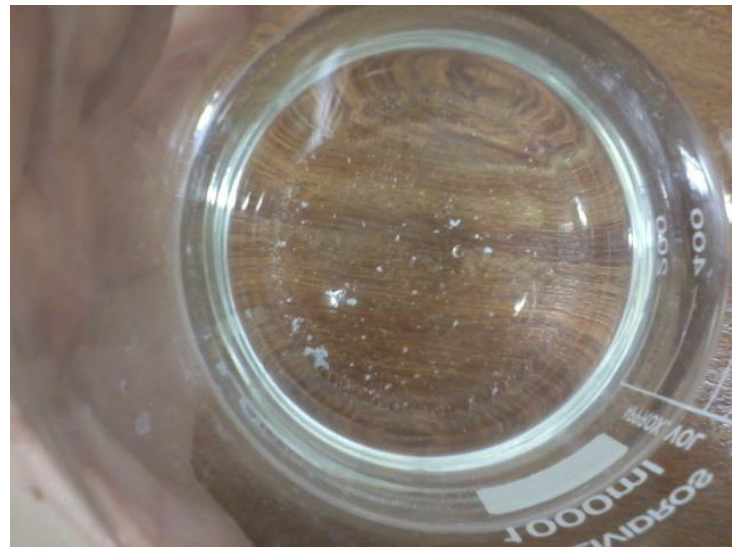

Figura 5: Particulados observados no final do processo da amostra 2B.

Os resultados dos testes de EIE são apresentados das Figuras 6 a 9, através do diagrama de Nyquist para as amostras com recobrimento para diferentes tempos de imersão em $\mathrm{NaCl} \mathrm{0,1} \mathrm{M}$. Avaliando os resulta dos de EIE é observado que os recobrimentos apresentam nas curvas de Nyquist dois arcos capacitivos, um em alta frequência (primeiro) que representa a capacitância da dupla camada e a resistência a transferência de carga entre o recobrimento e o eletrólito e outro em média/baixa frequência (segundo) que representa os processos corrosivos que ocorrem na interface substrato/recobrimento [16]. Sendo que o arco em alta frequência é menor que o segundo. Esse comportamento pode ser observado melhor na ampliação das curvas, Figuras 6 a 8. Com o tempo de imersão os arcos vão se tornando menores, o que representa uma diminuição na resistência à corrosão desses recobrimentos $[15,16]$.

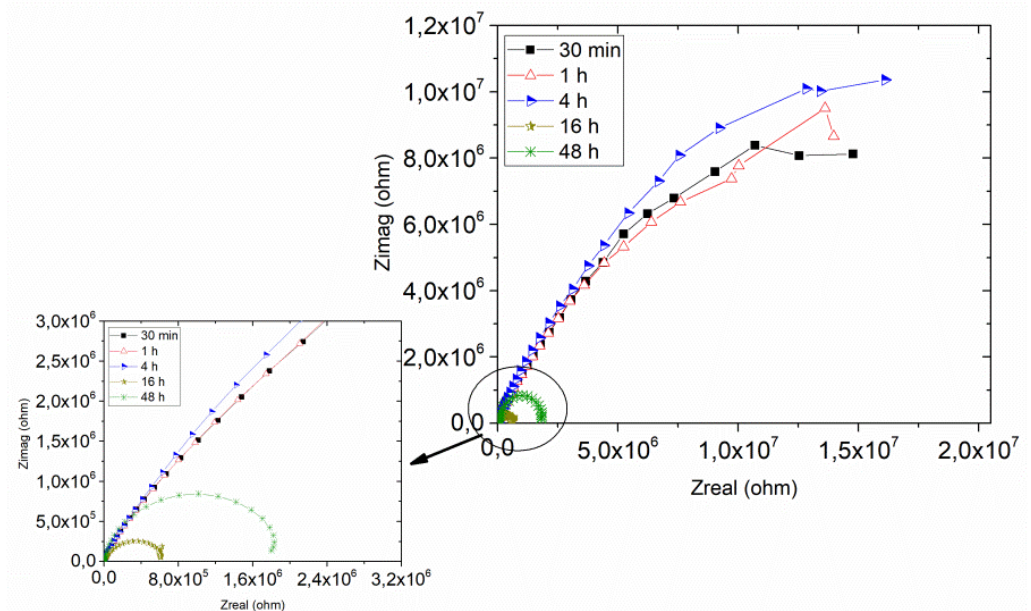

Figura 6: Resultado de EIS para a amostra $1 \mathrm{~A}$ em diferentes tempos de imersão em $\mathrm{NaCl} 0,1 \mathrm{M}$. 


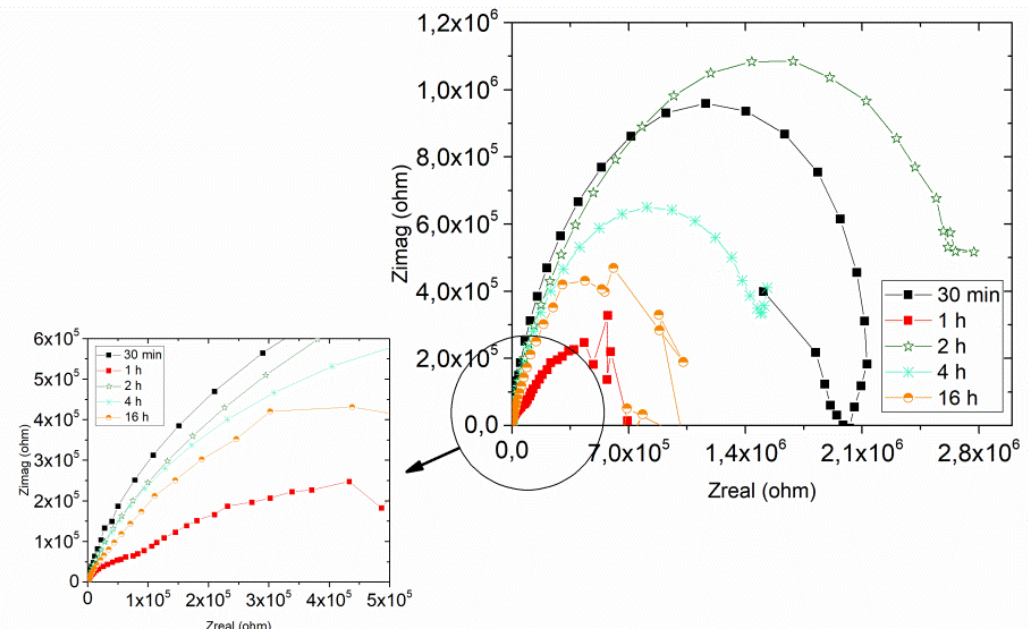

Figura 7: Resultado de EIS para a amostra 1B em diferentes tempos de imersão em $\mathrm{NaCl} 0,1 \mathrm{M}$.

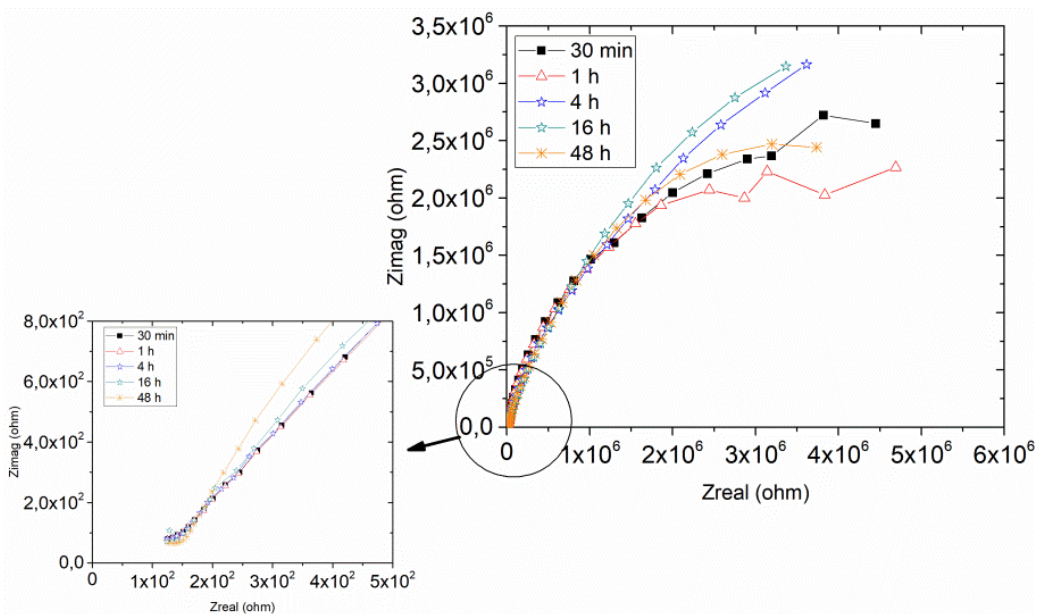

Figura 8: Resultado de EIS para a amostra 2A em diferentes tempos de imersão em $\mathrm{NaCl} 0,1 \mathrm{M}$.



Figura 9: Resultado de EIS para a amostra 2B em diferentes tempos de imersão em $\mathrm{NaCl} 0,1$ molar.

As curvas resultantes da EIE foram ajustadas através de circuito equivalente para uma melhor caracterização dos recobrimentos. O modelo de circuito elétrico equivalente utilizado para os ajustes está apresentado na Figura 10, onde R1 representa a resistência do eletrólito, CPE1 e R2 em paralelo representam a capacitância do filme passivo/recobrimento e a resistência de poro respectivamente, o par CPE2 e R3, em série com 
R2 estão associados à capacitância da dupla camada elétrica e à resistência de transferência de carga na interface metal/solução. O elemento de fase constate (CPE) foi empregado no lugar de um elemento capacitivo de forma a refletir todas as heterogeneidades, rugosidade e outras propriedades dielétricas não ideais do recobrimento [16].

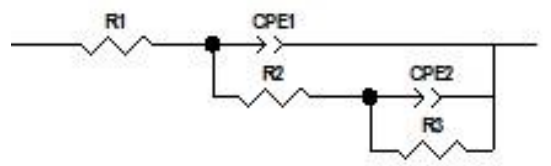

Figura 10: Modelo do circuito equivalente empregado no ajuste das curvas de EIE.

O ajuste dos resultados de EIE através do circuito elétrico apresentado na Figura 10 permite acompanhar a evolução dos elementos durante a imersão. A resistência R2, obtida em baixas frequências, é dita como a resistência a transferência de carga e pode ser considerada a principal resistência à corrosão do sistema [17]. Os resultados do ajuste para a resistência à transferência de carga e a capacitância do recobrimento, em função do tempo de imersão, são apresentados nas Figuras 11 e 12, respectivamente. Ao avaliar esses resultados, observa-se que os recobrimentos obtidos pelos procedimentos $1 \mathrm{~A}$ e $2 \mathrm{~A}$ são os que apresentaram maiores valores para essa resistência. Recobrimentos que apresentam uma camada interna mais compacta geralmente possuem maiores valores para $\mathrm{R} 2[14,16]$ e como mencionado anteriormente, a quantidade de recobrimentos obtidos para os procedimentos $1 \mathrm{~A}$ e $2 \mathrm{~A}$, sugerem que esses sejam mais compactos.

A evolução da capacitância do recobrimento com o tempo de imersão, observada na Figura 11, mostra pouca variação dos valores com o tempo de imersão, porém, pode-se dizer que os procedimentos $1 \mathrm{~A}, 1 \mathrm{~B}$ e 2B foram os que resultaram em recobrimentos com melhores comportamentos capacitivos.

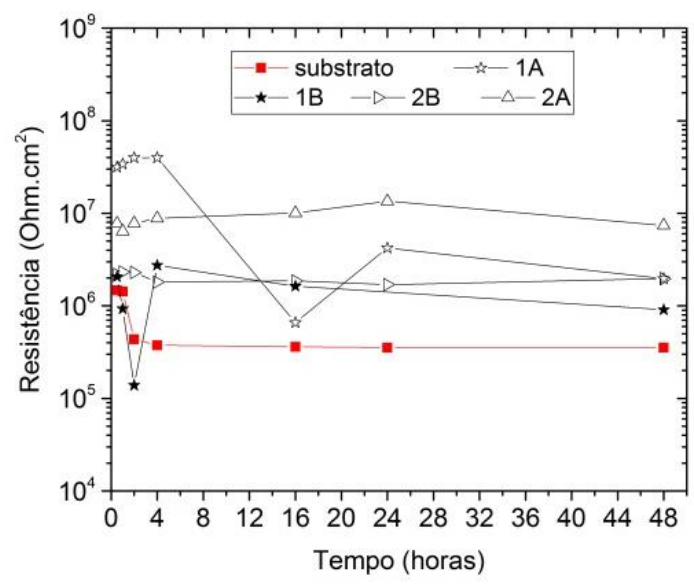

Figura 11: Resistência a transferência de carga (R3) em função do tempo de imersão para os diferentes recobrimentos. 


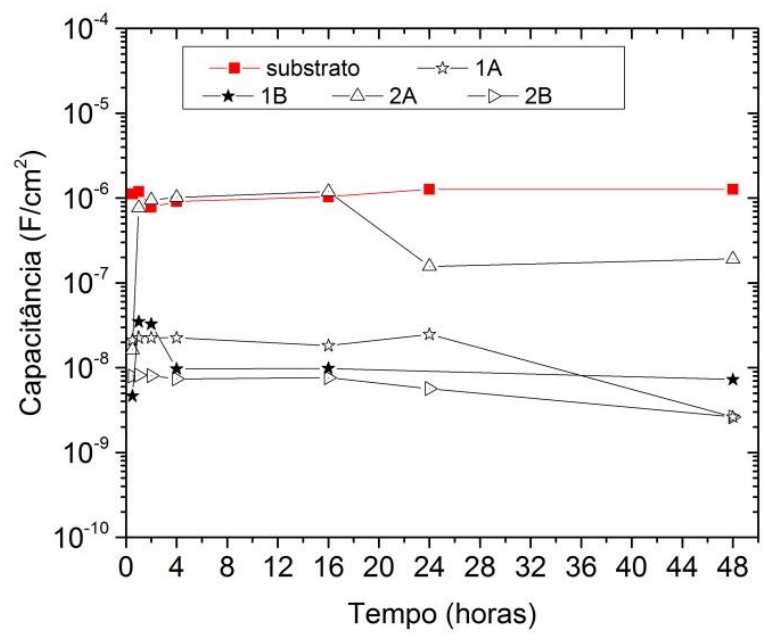

Figura 12: Capacitância do recobrimento (CPE1) em função do tempo de imersão para os diferentes recobrimentos.

\section{CONCLUSÕES}

Os recobrimentos formados a partir do processo de plasma eletrolítico resultaram em um melhor desempenho em termos de resistência à corrosão, observado através das análises de EIE, uma vez que apresentaram maiores valores de resistência na interface recobrimento/substrato. As amostras tratadas a $250 \mathrm{~V}$ apresentaram resultados melhores que a $300 \mathrm{~V}$, um dos motivos é pela maior densidade do recobrimento formado. Pelos resultados obtidos, pode-se dizer que os recobrimentos possuem porosidade e que com os procedimentos empregados não foi alcançado todos os estágios de formação. Mas mesmo assim, este filme já apresentou melhoras na proteção anticorrosiva do alumínio.

\section{AGRADECIMENTOS}

Os autores agradecem o suporte financeiro das agências FAPEMIG (projeto APQ-00190-10) e FAPEMA.

\section{BIBLIOGRAFIA}

[1] MELETIS, E.I., NIE, X., WANG, F.L., et al., "Electrolytic plasma processing for cleaning and metalcoating of steel surfaces", Surface and Coatings Technology, v.150, n. 2-3, pp. 246-256, Fev. 2002.

[2] WALSH, F. C., LOW, C. T. J., WOOD, R. J. K., et al., "Plasma electrolytic oxidation (PEO) for production of anodised coatings on lightweight metal (Al, Mg, Ti) alloys", The International Journal of Surface Engineering and Coatings,v. 87, n.3, pp. 122-135, 2009.

[3] RAJ, V., MUBARAK ALI, M., "Formation of ceramic alumina nanocomposite coatings on aluminium for enhanced corrosion resistance", Journal of Materials Processing Technology, v.209, n. 12-13, pp. 53415352, Jul. 2009.

[4] GUOHUA, L.V., WEICHAO, G., HUAN, C., W., et al., "Characteristic of ceramic coatings on aluminum by plasma electrolytic oxidation in silicate and phosphate electrolyte", Applied Surface Science, v. 253, n. 5 , pp. 2947-2952, Dez 2006.

[5] WU, H., WANG, J., LONG,B., et al., "Ultra-hard ceramic coatings fabricated through microarc oxidation on aluminium alloy", Applied Surface Science, v. 252, n.5, pp. 1545-1552, Dez. 2005.

[6] TASCHNER, C., LJUNGBERG, B., ALFREDSSON, V., et al., "Deposition of hard crystalline Al2O3 coatings by bipolar pulsed d.c. PACVD”, Surface and Coatings Technology, v. 109, pp. 257-264, Out. 1998.

[7] LIU, C., HE, D., YAN, Q., et al., "An investigation of the coating/substrate interface of plasma electrolytic oxidation coated aluminium", Surface \& Coatings Technology, v. 280, pp. 86-91, Out. 2015. 
[8] SNIZHKO, L.O, YEROKHIN, A.L, PILKINGTON, A., et al., "Anodic processes in plasma electrolytic oxidation of aluminium in alkaline solutions", Electrochimica Acta, v. 49, n. 13, pp. 2085-2095, Maio 2004.

[9] ARUNNELLAIAPPAN, T., BABU, N.K., KRISHNA, L. R., et al., "Influence of frequency and duty cycle on microstructure of plasma electrolytic oxidized AA7075 and the correlation to its corrosion behaviour", Surface \& Coatings Technology, v. 280, pp. 136-147, Out. 2015.

[10] WEI, T., YAN, F., TIAN J., "Characterization of wear and corrosion resistance of micro arc oxidation ceramic coatings on aluminum alloys", Journal of Alloys and Compounds, v.389, n. 1-2, pp.169-176, Marc. 2005.

[11] SRINIVASAN, P. B., BLAWERT, C., DIETZEL, W., "Dry sliding wear behaviour of plasma electrolytic oxidation coated AZ91 cast magnesium alloy", Wear, v. 266, n. 11 -12, pp.1241-1247, Maio 2009.

[12] MALAYOGLU U., TEKIN K.C., SHRESTHA S., "An investigation into the mechanical and tribological properties of plasma electrolytic oxidation and hard-anodized coatings on 6082 aluminum alloy", Material Science and Engineering A, v. 528, pp. 7451-7460, Set. 2011.

[13] HUSSEIN, R.O., NORTHWOOD, D.O., NIE, X., "The influence of pulse timing and current mode on the microstructure and corrosion behaviour of a plasma electrolytic oxidation (PEO) coated AM60B magnesium alloy", Journal of Alloys and Compounds, v. 541, pp. 41-48, Nov. 2012.

[14] SONGA, G., SHI, Z., "Corrosion mechanism and evaluation of anodized magnesium alloys" Corrosion Science, v. 85, pp.126-140, Ag. 2014.

[15] LIU, C., LIU, P., HUANG, Z., et al., "The correlation between the coating structure and the corrosion behaviour of the plasma electrolytic oxidation coating on aluminium", Surface \& Coatings Technology, v. 286, pp. 223-230, Jan. 2016.

[16] WEN, L., WANG,Y., ZHOU, Y., et al., "Corrosion evaluation of microarc oxidation coatings formed on 2024 aluminium alloy”, Corrosion Science, v. 52, n. 8, pp. 2687-2696, Ag. 2010.

[17] CURRAN, J.A., CLYNE, T.W., "Thermo physical properties of plasma electrolytic oxidation on aluminium, Surface and Coating Technology, v. 199, p.168-176, Set. 2005.

[18] YEROKHIN, A.L., NIE, X., LEYLAND, A., et al., "Plasma electrolysis for surface engineering", Surface and Coatings Technology, v.122, n. 2-3, pp. 73-93, Dez.1999

[19] GU, W., LV, G., CHEN, H., et al., "Characterisation of ceramic coatings produced by plasma electrolytic oxidation of aluminium alloy", Materials Science and Engineering A, v. 447, n. 1-2, pp.158-162, Fev. 2007.

[20] KUMARI, R., BLAWERT, C., MAJUMDAR, J. D., "Microstructures and Properties of Plasma Electrolytic Oxidized Ti Alloy (Ti-6Al-4V) for Bio-implant Application”, Metallurgical And Materials Transactions A, v. 47A, pp. 788-800, Fev. 2016.

[21] WANG, Y., JIANG, Z., YAO, Z., et al., "Microstructure and corrosion resistance of ceramic coating on carbon steel prepared by plasma electrolytic oxidation", Surface \& Coatings Technology, v. 204, n. 11, pp. 1685-1688, Fev. 2010.

[22] HWANG, I. J., HWANG, D. Y., KIM, Y. M., et al., "Formation of uniform passive oxide layers on high $\mathrm{Si}$ content Al alloy by plasma electrolytic oxidation", Journal of Alloys and Compounds, v. 504, s.1, pp. S527-S530, Ag. 2010.

[23] WANG, Y.K., SHENG, L., XIONG, R.Z., et al., "Effects of additives in electrolyte on characteristics of ceramic coatings formed by microarc oxidation", Surface Engineering, v. 15, n.2, pp. 109-111,1999.

[24] GUPTA, P., TENHUNDFELD, G., DAIGLE, E.O., et al., "Electrolytic plasma technology: Science and engineering-An overview", Surface \& Coatings Technology, v.201, pp. 8746-8760, 2007.

\section{ORCID}

Maria Eliziane Pires de Souza

Bruna Almeida Barbosa

Giselle Barata Costa https://orcid.org/0000-0002-9184-3921

https://orcid.org/0000-0003-2777-1078

https://orcid.org/0000-0003-2339-8755 